\title{
COVID-19 and your rheumatology patients
}

\section{Posted April 18, 2020}

\section{ABSTRACT}

The COVID-19 pandemic has caused a sense of unease in the rheumatology community about the risk to patients with immune-mediated diseases, given their predisposition to infection due to underlying disease states and immunosuppressive therapies. Thus far, there have been few cases of COVID-19 reported in patients with immune-mediated diseases and there have been no formal guidelines released on the management of patients with immune-mediated diseases in the setting of COVID-19. Results of clinical trials and data from the Global Rheumatology Alliance registry are eagerly awaited to gain further insight into the impact of this novel infection on our vulnerable patient population.

\section{RHEUMATOLOGY AND COVID-19}

Since the start of the COVID-19 pandemic, there has been a growing sense of unease in the rheumatology community about the risk to our patients with immunemediated diseases, given their predisposition to infection due to underlying disease states and immunosuppressive therapies. It's only natural for us to assume that patients with immune-mediated diseases will be at increased risk of COVID-19 infection and its complications. However, there are essentially no data to suggest this to date. Results of a European experience in Lombardy, Italy, with COVID-19-positive rheumatic disease patients were reported in a very small numbers. ${ }^{1}$ Compared with other regions of Italy, Lombardy has seen the highest incidence of COVID-19, with almost 55,000 cases reported. Monti et al circulated a survey to patients with chronic arthritis treated with conventional or biologic disease-modifying antirheumatic drugs, and out of 320 responses, 4 patients had confirmed COVID-19, and 4 had probable infection.

The statements and opinions expressed in COVID-19 Curbside Consults are based on experience and the available literature as of the date posted. While we try to regularly update this content, any offered recommendations cannot be substituted for the clinical judgment of clinicians caring for individual patients.

doi:10.3949/ccjm.87a.ccc027
One patient required hospital admission for supplemental oxygen, and all recovered without incident.

Of course these findings in no way allow us to draw conclusions about the incidence of COVID-19 in patients with immune-mediated diseases or the clinical outcomes. Yet they add to our observation that thus far, there have been few cases of COVID-19 reported in patients with immune-mediated diseases. Interestingly, these authors also observed that out of 700 patients hospitalized in the ICU with severe COVID-19, none were receiving conventional or biologic disease-modifying drugs at baseline. ${ }^{1}$ A single case-series of 21 patients with COVID-19 at Evergreen Hospital in Kirkland, WA, included 1 patient with "rheumatologic disease," and 2 with a history of solid-organ transplant. ${ }^{2}$ So far, this is quite different than the influenza experience in patients with immune-mediated diseases, where it is well known that immunosuppressed patients have a greater risk of influenza, morbidity from influenza, and prolonged viral shedding. ${ }^{3,4}$

While many questions remain unanswered surrounding the risk of COVID-19 in rheumatology patients, the above observations and anecdotes serve to reinforce how we counsel our patients during this pandemic. Many patients wonder if they should stop their immunosuppression, or hold off on starting or switching to a new biologic due to fear of COVID19. At the time of this publication, there have been no formal guidelines released on the management of patients with immune-mediated diseases in the setting of COVID-19. However, the American College of Rheumatology has released suggestions for clinical decision-making that support keeping scheduled infusion appointments and making decisions regarding intra-articular joint injections on a case-by-case basis, as well as recommendations regarding telemedicine. ${ }^{5}$

We recommend that patients continue to present for scheduled biologic infusions, as missing or postponing these appointments could lead to disease flare, requirement for increasing doses of glucocorticoids, 
possible increased exposure to the healthcare setting or even hospitalization. Of course, these decisions should be made on a case-by-case basis. For example, intravenous abatacept infusion for a rheumatoid arthritis patient whose disease is in remission may be less urgent than for a patient with granulomatosis with polyangiitis who is due for rituximab.

Many would categorize infusion medications for osteoporosis treatment as "nonessential" during COVID-19; however, we should approach denosumab differently, as fracture risk increases rapidly after discontinuation of this drug. ${ }^{6}$ Regardless of COVID-19, it is recommended that patients discontinuing denosumab be rapidly transitioned to an antiresporptive agent. ${ }^{6}$ Thus, at present, patients unable to present for denosumab should be started on an oral bisphosphonate for the short-term. Alternatively, if the patient has a healthcare provider in the family, the drug could potentially be shipped to the patient's home for administration.

We also recommend that patients continue their immunosuppressive medications and not stop them pre-emptively, and this is aligned with other expert opinion. ${ }^{7,8}$ Hydroxychloroquine, used frequently in the treatment of systemic lupus erythematosus, rheumatoid arthritis, and other immune-mediated diseases, is now under the spotlight as a treatment candidate for COVID-19, based on limited and inconclusive data. ${ }^{9}$ Due to drug shortages, we now find our patients having difficulty filling their prescriptions. In these scenarios, and given the long terminal half-life of the drug, I have been recommending that patients, based on their disease and dependency on the drug, to decrease their daily dose by half, to extend the supply of their current prescription; we feel this is safe for short periods (1-2 months or more). This would be more appropriate for rheumatoid arthritis and or mild systemic lupus erythematosus, and less appropriate for lupus patients with significant end-organ involvement. In my opinion, this is an imperfect solution but a reasonable compromise. Hopefully, drug availability will not be an ongoing issue as production is being ramped up and regulatory steps are being taken to protect our patients, albeit in limited fashion.

The rheumatology community has responded rapidly to COVID-19, by forming the COVID-19 Global Rheum Alliance (rheum-covid.org), an international effort with a goal to aid clinicians and researchers in real time as the pandemic evolves, providing insights into how COVID-19 is impacting patients with rheumatic disease. In early March, 2020, the concept was developed and publicized through social media, e-mail, listservs, and personal and professional networks. Within 2 days of the initial idea, the alliance engaged rheumatologists across the world, with support from more than 100 professional and nonprofit organizations, including the American College of Rheumatology, European League Against Rheumatism, British Society of Rheumatology, and many others. Early descriptive data are updated frequently online and through Twitter (https://mailchi.mp/ a6dc80671481/just-launched-the-covid-19-globalrheumatology-registry-4810762), and we anxiously await formal analysis of these data.

Many questions remain for the rheumatologic community: What is the risk of COVID-19 in patients with immune-mediated diseases? Will baseline immunosuppression affect risk or outcomes of COVID-19? What therapies will be effective? As rheumatologists, we find ourselves well-positioned to assist in managing COVID-19, as many targeted therapies we use to treat immune-mediated diseases are candidates for treatment of COVID-19 (anti-IL-1, IL-6, TNF, to name a few). We anxiously await results of clinical trials and data from the Global Rheumatology Alliance registry to gain further insight into the impact of this novel infection on our vulnerable patient population.

\section{REFERENCES}

1. Monti S, Balduzzi S, Delvino P, Bellis E, Quadrelli VS, Montecucco C. Clinical course of COVID-19 in a series of patients with chronic arthritis treated with immunosuppressive targeted therapies. Ann Rheum Dis 2020; Apr 2. doi:10.1136/annrheumdis-2020-217424

2. Arentz M, Yim E, Klaff L, et al. Characteristics and outcomes of 21 critically ill patients with COVID-19 in Washington State. JAMA 2020; Mar 19. doi:10.1001/jama.2020.4326

3. Blumentals WA, Arreglado A, Napalkov P, Toovey S. Rheumatoid arthritis and the incidence of influenza and influenza-related complications: a retrospective cohort study. BMC Musculoskelet Disord 2012; 13:158. doi:10.1186/1471-2474-13-158

4. Memoli MJ, Athota R, Reed S, et al. The natural history of influenza infection in the severely immunocompromised vs nonimmunocompromised hosts. Clin Infect Dis 2014; 58(2):214-224. doi:10.1093/cid/ cit725

5. American College of Rheumatology. Guiding principles from the American College of Rheumatology for decision making around in person urgent versus virtual non urgent medical care. https://www. rheumatology.org/Portals/0/Files/Guiding-Principles-Urgent-vs-NonUrgent-Services.pdf. Accessed April 14, 2020.

6. Cummings SR, Ferrari S, Eastell R, et al. Vertebral fractures after discontinuation of denosumab: a post hoc analysis of the randomized placebo-controlled FREEDOM trial and its extension. J Bone Miner Res 2018; 33(2):190-198. doi:10.1002/jbmr.3337

7. Ferro F, Elefante $\mathbf{E}$, Baldini $\mathbf{C}$, et al. COVID-19: the new challenge for rheumatologists. Clin Exp Rheumatol 2020; 38(2):175-180. pmid:32207680

8. Winthrop KL. Who needs a Corona? Arthritis Rheumatol 2020; Mar 17. doi:10.1002/art.41260

9. Gautret P, Lagier JC, Parola P, et al. Hydroxychloroquine and azithromycin as a treatment of COVID-19: results of an open-label non-randomized clinical trial. Int J Antimicrob Agents 2020; Mar 20. doi:10.1016/j.ijantimicag.2020.105949 